\title{
Toeplitz Operators on Dirichlet-Type Space of Unit Ball
}

\author{
Jin Xia, Xiaofeng Wang, and Guangfu Cao \\ School of Mathematics and Information Science and Key Laboratory of Mathematics and Interdisciplinary Sciences of \\ the Guangdong Higher Education Institute, Guangzhou University, Guangzhou 510006, China
}

Correspondence should be addressed to Xiaofeng Wang; wangxiaofeng514@hotmail.com

Received 10 March 2014; Accepted 25 May 2014; Published 22 June 2014

Academic Editor: Kunquan Lan

Copyright (c) 2014 Jin Xia et al. This is an open access article distributed under the Creative Commons Attribution License, which permits unrestricted use, distribution, and reproduction in any medium, provided the original work is properly cited.

We construct a function $u$ in $L^{2}\left(\mathbb{B}_{n}, d V\right)$ which is unbounded on any neighborhood of each boundary point of $\mathbb{B}_{n}$ such that Toeplitz operator $T_{u}$ is a Schatten $p$-class $(0<p<\infty)$ operator on Dirichlet-type space $\mathscr{D}\left(\mathbb{B}_{n}, d V\right)$. Then, we discuss some algebraic properties of Toeplitz operators with radial symbols on the Dirichlet-type space $\mathscr{D}\left(\mathbb{B}_{n}, d V\right)$. We determine when the product of two Toeplitz operators with radial symbols is a Toeplitz operator. We investigate the zero-product problem for several Toeplitz operators with radial symbols. Furthermore, the corresponding commuting problem of Toeplitz operators whose symbols are of the form $\xi^{k} u$ is studied, where $k \in \mathbb{Z}^{n}, \xi \in \partial \mathbb{B}_{n}$, and $u$ is a radial function.

\section{Introduction}

Let $\mathbb{B}_{n}$ represent the open unit ball in several complex spaces $\mathbb{C}^{n}$. The Sobolev space $L^{2,1}\left(\mathbb{B}_{n}, d V\right)$ consists of the functions which satisfy

$$
\|f\|_{2,1}=\left(\sum_{i=1}^{n} \int_{\mathbb{B}_{n}}\left(\left|\frac{\partial f}{\partial z_{i}}(z)\right|^{2}+\left|\frac{\partial f}{\partial \bar{z}_{i}}(z)\right|^{2}\right) d V\right)^{1 / 2}<\infty,
$$

where $d A$ denotes the normalized Lebesgue volume measure on $\mathbb{B}_{n} . L^{2,1}\left(\mathbb{B}_{n}\right)$ is a Hilbert space with inner product

$$
\langle f, g\rangle_{2,1}=\sum_{i=1}^{n}\left\langle\frac{\partial f}{\partial z_{i}}, \frac{\partial g}{\partial z_{i}}\right\rangle+\sum_{i=1}^{n}\left\langle\frac{\partial f}{\partial \bar{z}_{i}}, \frac{\partial g}{\partial \bar{z}_{i}}\right\rangle,
$$

where $\langle\cdot, \cdot\rangle$ denotes the inner product in $L^{2}\left(\mathbb{B}_{n}, d V\right)$. The Dirichlet-type space $\mathscr{D}$ is the subspace of all analytic functions $g$ in $L^{2,1}\left(\mathbb{B}_{n}\right)$ with $g(0)=0$. Then, $\mathscr{D}$ is a closed subspace of the $L^{2,1}\left(\mathbb{B}_{n}\right)$. Let $P$ be the orthogonal projection from $L^{2,1}\left(\mathbb{B}_{n}\right)$ onto $\mathscr{D} . P$ is an integral operator represented by

$$
P(f)(w)=\langle f, K(z, w)\rangle_{2,1}=\sum_{i=1}^{n} \int_{\mathbb{B}_{n}} \frac{\partial f}{\partial z_{i}} \frac{\overline{\partial K_{w}}}{\partial z_{i}} d V(z),
$$

where $K_{w}(z)=K(z, w)$ is the reproducing kernel of $\mathscr{D}$. By computation, we know

$$
K(z, w)=\sum_{\alpha \in \mathbb{N}^{n}-\{(0, \ldots, 0)\}} \frac{(|\alpha|+n-1) !}{|\alpha| n ! \alpha !} z^{\alpha} \bar{w}^{\alpha}
$$

where $\alpha=\left(\alpha_{1}, \ldots, \alpha_{n}\right) \in \mathbb{N}^{n}, \alpha !=\alpha_{1} ! \cdots \alpha_{n} !, z^{\alpha}=z_{1}^{\alpha_{1}} \cdots z_{n}^{\alpha_{n}}$, and $|\alpha|=\sum_{i=1}^{n} \alpha_{i}$.

In recent years, the Dirichlet-type space has received a lot of attention from mathematicians in the areas of modern analysis and probability and statistical analysis. Many mathematicians are interested in function theory and operator theory on the Dirichlet-type space (See $[1,2])$. In [3, 4], for the Dirichlet-type space of one complex variable, that is, $n=1$, Rochberg and Wu defined the Toeplitz operator with nonnegative measure $\mu$ on $\mathbb{B}_{1}$ as follows: $T_{\mu}: \mathscr{D} \rightarrow \mathscr{D}$ by

$$
T_{\mu}(f)(w)=\int_{\mathbb{B}_{1}} f(z) \overline{K(z, w)} d \mu(z)
$$

Rochberg and Wu discussed the boundedness and compactness. Lu and Sun define Toeplitz operators on a Dirichlet-type space of several variables in [5]. 
Definition 1. Suppose that $\mu$ is a finite measure on $\mathbb{B}_{n}$. Toeplitz operators on the Dirichlet-type space with symbol $\mu$ are defined as follows:

$$
T_{\mu}(f)(w)=\int_{\mathbb{B}_{n}} f(z) \overline{K(z, w)} d \mu(z), \quad f \in \mathscr{D} .
$$

If $d \mu=\varphi d A, \varphi \in L^{1}\left(\mathbb{B}_{n}, d A\right)$, we write $T_{\mu}=T_{\varphi}$ and $T_{\varphi}$ is called the Toeplitz operator with symbol $\varphi$.

\section{Schatten $p$-Class Toeplitz Operators with Unbounded Symbols}

In general, if $u \notin L^{\infty}\left(\mathbb{B}_{n}\right)$, the space of essentially bounded functions on $\mathbb{B}_{n}$ and then $T_{u}$ is densely defined only. In the case of Hardy space, it is well known that $T_{u}$ is bounded if and only if $u$ is essentially bounded, and $T_{u}$ is compact if and only if $u=0$ (see Douglas [6] and Davie and Jewell [7]). However, there are indeed bounded and compact Toeplitz operators with unbounded symbols on Bergman spaces of one complex variable; in fact, Miao and Zheng [8] have introduced a class of functions, called BT, which contains $L^{\infty}$, for $u \in \mathrm{BT}$; $T_{u}$ is compact on $L_{a}^{2}$ if and only if the Berezin transform of $T_{u}$ vanishes on the unit circle. Zorboska [9] has proved that if $u$ belongs to the hyperbolic BMO space, the $T_{u}$ is compact if and only if the Berezin transform of $u$ vanishes on the unit circle. Cima and Cuckovic [10] construct a class of unbounded functions over a Cantor set; the Toeplitz operators with these functions are compact. Essentially, if the values of the function $T_{u}$ vanish rapidly near the unit circle in the sense of measure $d V$, then $T_{u}$ will be compact. Cao [11] also construct compact Toeplitz operators and trace class Toeplitz operators with unbounded symbols on Bergman space of several complex variables.

In this section, we construct the compact Toeplitz operators and the Schatten $p$-class Toeplitz operators for $0<$ $p<\infty$ with unbounded symbols on Dirichlet-type space of several complex variables.

For preparation, we introduce a special set in $\mathbb{B}_{n}$, which is important for building our main results in this section.

For $a>0$ and $\xi \in \partial \mathbb{B}_{n}$, the boundary of $\mathbb{B}_{n}$, let

$$
\begin{aligned}
& D(\xi, a)=\left\{z \in \mathbb{B}_{n}:\right. {\left[1-(1-|z|)^{a}\right]^{1 / 2} \cdot|z-\xi| } \\
& \leq|\operatorname{Re}\langle\xi, z-\xi\rangle|, \\
&\operatorname{Re}\langle z, \xi\rangle>0\} ;
\end{aligned}
$$

then it is obvious that $D(\xi, a)$ is a domain in $\mathbb{B}_{n}$, which is called circular-like cone with vertex $\xi$, because it looks like a circular cone. For $0<r<1$, set $\mathbb{B}_{n}(r)=\left\{z \in \mathbb{C}_{n}:|z|<r\right\}$ to be the ball with radius $r$. We use $d S_{r}$ to denote the area measure on $\partial \mathbb{B}_{n}(r)$, for $r=1 ; d S_{1}=d S$ is the normalized area measure on $\partial \mathbb{B}_{n}$; then there holds $S_{r}\left(\partial \mathbb{B}_{n}(r)\right)=O\left(r^{2 n-1}\right)$ and $S_{1}\left(\partial \mathbb{B}_{n}\right)=1$. For $b>0$, because of the property of $D(\xi, a)$, there exits proper $a(b)>0$ such that, for any $0<r<1$,

$$
S_{r}\left(D(\xi, a(b)) \cap \partial \mathbb{B}_{n}(r)\right)<\lambda\left(1-r^{2}\right)^{b},
$$

where $\lambda$ is a constant number that is independent of $\xi$ and $r$. For convenience, we write $D_{b}(\xi)=D(\xi, a(b))$.
Lemma 2 (Poincaré's inequality). Suppose that $1 \leq p<\infty$, $\Omega$ is a bounded domain in $\mathbb{R}^{n}$ and $u$ belongs to Sobolev space $W_{0}^{1, p}(\Omega)$; then

$$
\int_{\Omega}|u|^{p} d V \leq C \int_{\Omega}|\nabla u|^{p} d V
$$

where $C$ is a constant that depends on $\Omega$.

Lemma 3. Suppose that $c>0, U_{c}(z)=\left(1-|z|^{2}\right)^{-c}$, and $z \in \mathbb{B}_{n}$. For any $\xi \in \mathbb{B}_{n}$, let $b \geq n+2 c+2$; we use $\chi_{D_{b}(\xi)}(z)$ to represent the characteristic function of the set $D_{b}(\xi)$. Then, $u(z)=\chi_{D_{b}(\xi)}(z) U_{c}(z)$ introduces a compact Toeplitz operator $T_{\varphi}$ on $\mathscr{D}$.

Proof. Take a sequence $\left\{f_{k}(z)\right\}_{k}$ from $\mathscr{D}$ such that $f_{k} \rightarrow 0$ weakly and $\left\|f_{k}\right\|_{2,1} \leq 1$; it is enough to obtain that

$$
\left\|T_{u} f_{k}\right\|_{2,1} \longrightarrow 0
$$

From Definition 1, we find that

$$
\begin{aligned}
& \left\|T_{u} f_{k}(z)\right\|_{2,1}^{2} \\
& =\sum_{i=1}^{n} \int_{\mathbb{B}_{n}}\left|\int_{\mathbb{B}_{n}} u(w) f_{k}(w) \frac{\partial K(z, w)}{\partial z_{i}} d V(w)\right|^{2} d V(z) \\
& =\sum_{i=1}^{n} \int_{\mathbb{B}_{n}}\left|\int_{D_{b}(\xi)} \frac{f_{k}(w)}{\left(1-|w|^{2}\right)^{c}} \frac{\partial K(z, w)}{\partial z_{i}} d V(w)\right|^{2} d V(z) .
\end{aligned}
$$

For $m \in(0,1)$, set $D_{b}(\xi, m)=\left\{z \in D_{b}(\xi):|z|>m\right\}$; then

$$
\begin{gathered}
\sum_{i=1}^{n} \int_{\mathbb{B}_{n}}\left|\int_{D_{b}(\xi)} \frac{f_{k}(w)}{\left(1-|w|^{2}\right)^{c}} \frac{\partial K(z, w)}{\partial z_{i}} d V(w)\right|^{2} d V(z) \\
\leq 2 \sum_{i=1}^{n} \int_{\mathbb{B}_{n}}\left|\int_{D_{b}(\xi, m)} \frac{f_{k}(w)}{\left(1-|w|^{2}\right)^{c}} \frac{\partial K(z, w)}{\partial z_{i}} d V(w)\right|^{2} d V(z) \\
+2 \sum_{i=1}^{n} \int_{\mathbb{B}_{n}} \mid \int_{D_{b}(\xi)-D_{b}(\xi, m)} \\
\times\left.\frac{f_{k}(w)}{\left(1-|w|^{2}\right)^{c}} \frac{\partial K(z, w)}{\partial z_{i}} d V(w)\right|^{2} d V(z) .
\end{gathered}
$$


By Hölder's and Poincaré's inequality,

$$
\begin{aligned}
& \mid \int_{D_{b}(\xi, m)}\left.\frac{f_{k}(w)}{\left(1-|w|^{2}\right)^{c}} \frac{\partial K(z, w)}{\partial z_{i}} d V(w)\right|^{2} \\
& \leq \int_{D_{b}(\xi, m)}\left|f_{k}(w)\right|^{2} d V(w) \\
& \quad \times \int_{D_{b}(\xi, m)} \frac{1}{\left(1-|w|^{2}\right)^{2 c}}\left|\frac{\partial K(z, w)}{\partial z_{i}}\right|^{2} d V(w) \\
& \leq C \int_{D_{b}(\xi, m)} \frac{1}{\left(1-|w|^{2}\right)^{2 c}}\left|\frac{\partial K(z, w)}{\partial z_{i}}\right|^{2} d V(w),
\end{aligned}
$$

where $C$ is constant. Then

$$
\begin{aligned}
& \sum_{i=1}^{n} \int_{\mathbb{B}_{n}}\left|\int_{D_{b}(\xi, m)} \frac{f_{k}(w)}{\left(1-|w|^{2}\right)^{c}} \frac{\partial K(z, w)}{\partial z_{i}} d V(w)\right|^{2} d V(z) \\
& \leq C \sum_{i=1}^{n} \int_{\mathbb{B}_{n}} \int_{D_{b}(\xi, m)} \frac{1}{\left(1-|w|^{2}\right)^{2 c}}\left|\frac{\partial K(z, w)}{\partial z_{i}}\right|^{2} d V(w) d V(z) \\
& =C \int_{D_{b}(\xi, m)} \frac{1}{\left(1-|w|^{2}\right)^{2 c}} d V(w) \\
& \times \int_{\mathbb{B}_{n}} \sum_{i=1}^{n}\left|\frac{\partial K(z, w)}{\partial z_{i}}\right|^{2} d V(z) \\
& =C \int_{D_{b}(\xi, m)} \frac{K(w, w)}{\left(1-|w|^{2}\right)^{2 c}} d V(w) \\
& =C \int_{D_{b}(\xi, m)}\left(\left(\sum_{\alpha \in \mathbb{N}^{n}-\{(0, \ldots, 0)\}}\right.\right. \\
& \left.\times \frac{(|\alpha|+n-1) !}{|\alpha| n ! \alpha !}|w|^{2 \alpha}\right) \\
& \left.\times\left(\left(1-|w|^{2}\right)^{2 c}\right)^{-1}\right) d V(w) \\
& \leq C \int_{D_{b}(\xi, m)} \frac{\sum_{\alpha \in \mathbb{N}^{n}}((|\alpha|+n) ! / n ! \alpha !)|w|^{2 \alpha}}{\left(1-|w|^{2}\right)^{2 c}} d V(w) \\
& =C \int_{D_{b}(\xi, m)} \frac{1}{\left(1-|w|^{2}\right)^{n+1+2 c}} d V(w) \\
& =2 n C \int_{0}^{1} r^{2 n-1} d r \\
& \times \int_{\partial \mathbb{B}_{n}} \chi_{D_{b}(\xi, m)}(r \eta) \frac{1}{\left(1-r^{2}\right)^{n+1+2 c}} d S(\eta) \\
& =c_{0} \int_{m}^{1} r^{2 n-1} d r
\end{aligned}
$$

$$
\begin{aligned}
& \times \int_{\partial \mathbb{B}_{n}(r) \cap D_{b}(\xi, m)} \frac{1}{\left(1-r^{2}\right)^{n+1+2 c}} d S(\eta) \\
= & c_{0} \int_{0}^{1} d r \int_{\partial \mathbb{B}_{n}(r) \cap D_{b}(\xi, m)} \frac{1}{\left(1-r^{2}\right)^{n+1+2 c}} d S_{r}(\eta) \\
\leq & \frac{c_{0}}{m} \int_{m}^{1} \int_{\partial \mathbb{B}_{n}(r) \cap D_{b}(\xi, m)} \frac{1}{\left(1-r^{2}\right)^{n+1+2 c}} d\left(r^{2}\right) \\
\leq & \frac{c_{1}}{m} \int_{m}^{1} \frac{\left(1-r^{2}\right)^{b}}{\left(1-r^{2}\right)^{n+1+2 c}} d\left(r^{2}\right) \\
= & \frac{c_{1}}{m} \frac{\left(1-m^{2}\right)^{b-n-2 c}}{b-n-2 c} \leq \frac{c_{1}}{m}\left(1-m^{2}\right)^{2},
\end{aligned}
$$

where $c_{0}, c_{1}$ are constants. Thus, for any $\varepsilon>0$, there exists a $m_{0} \in(0,1)$ such that

$$
\sum_{i=1}^{n} \int_{\mathbb{B}_{n}}\left|\int_{D_{b}(\xi, m)} \frac{f_{k}(w)}{\left(1-|w|^{2}\right)^{c}} \frac{\partial K(z, w)}{\partial z_{i}} d V(w)\right|^{2} d V(z) \leq \varepsilon
$$

for $m \in\left[m_{0}, 1\right)$.

On the other hand, because $D_{b}(\xi)-D_{b}(\xi, m) \subset \overline{\mathbb{B}_{n}\left(m_{0}\right)}$, the sequence $f_{k}$ converges uniformly to 0 in $\overline{\mathbb{B}_{n}\left(m_{0}\right)}$ by its weak convergence (see Zhu [12]). Then, for any $\varepsilon>0$, there is a $K_{0}$ such that, for any $k>K_{0}$, we find that $\left|f_{k}(w)\right|<\varepsilon$, $w \in D_{b}(\xi)-D_{b}(\xi, m)$. Thus

$$
\begin{aligned}
& \left|\int_{D_{b}(\xi)-D_{b}\left(\xi, m_{0}\right)} \frac{f_{k}(w)}{\left(1-|w|^{2}\right)^{c}} \frac{\partial K(z, w)}{\partial z_{i}} d V(w)\right| \\
& \quad \leq \varepsilon \int_{D_{b}(\xi)-D_{b}\left(\xi, m_{0}\right)} \frac{1}{\left(1-|w|^{2}\right)^{c}}\left|\frac{\partial K(z, w)}{\partial z_{i}}\right| d V(w) \\
& \quad \leq \varepsilon \frac{1}{\left(1-\left|m_{0}\right|^{2}\right)^{c}} \sum_{\alpha \in \mathbb{Z}^{+n}} \frac{(|\alpha|+n-1) !}{n ! \alpha !} m_{0}^{\alpha} .
\end{aligned}
$$

This shows that $\left\|T_{u} f_{k}\right\|_{2,1} \rightarrow 0$.

Using Lemma 3, we can construct a compact Toeplitz operator with a symbol that is unbounded on any neighborhood of every point in unit surface.

Theorem 4. There exists a function $u \in L^{1}\left(\mathbb{B}_{n}, d A\right)$ which is unbounded on any neighborhood of every point in unit surface (i.e., for any $\xi \in \partial \mathbb{B}_{n}$ and $r>0$,

$$
\underset{z \in \mathbb{B}_{n} \cap \mathbb{B}(\xi, r)}{\operatorname{essup}}|u(z)|=\infty,
$$

where $\mathbb{B}(\xi, r)=\{z:|z-\xi|<r\}) ; T_{u}$ is a compact operator on D.

Proof. Suppose that $c>0, b \geq n+2 c+2$, and $U_{c}(z)$ is the function defined in Lemma 3. Let $\left\{\xi_{i}\right\}_{i=1}^{\infty}$ be a countable dense 
subset of $\partial \mathbb{B}_{n}$; then, for every $\xi_{i}$, the $u_{i}=\chi_{D_{b}\left(\xi_{i}\right)} U_{c}$ introduces a compact Toeplitz operator $T_{u_{i}}$ by Lemma 3. Furthermore, for any $f \in \mathscr{D}$, by Hölder's and Poincarè's inequality,

$$
\begin{aligned}
& \left\|T_{u_{i}} f\right\|_{2,1}^{2} \\
& =\sum_{i=1}^{n} \int_{\mathbb{B}_{n}}\left|\int_{\mathbb{B}_{n}} u_{i}(w) f(w) \frac{\partial K(z, w)}{\partial z_{i}} d V(w)\right|^{2} d V(z) \\
& =\sum_{i=1}^{n} \int_{\mathbb{B}_{n}}\left|\int_{D_{b}\left(\xi_{i}\right)} \frac{f(w)}{\left(1-|w|^{2}\right)^{c}} \frac{\partial K(z, w)}{\partial z_{i}} d V(w)\right|^{2} d V(z) \\
& \leq\|f\|_{2}^{2} \sum_{i=1}^{n} \int_{\mathbb{B}_{n}} \int_{D_{b}\left(\xi_{i}\right)} \frac{\left|\partial K(z, w) / \partial z_{i}\right|^{2}}{\left(1-|w|^{2}\right)^{2 c}} d V(w) d V(z) \\
& \leq C\|f\|_{2,1}^{2} \int_{D_{b}\left(\xi_{i}\right)} \frac{1}{\left(1-|w|^{2}\right)^{2 c}} d V(w) \\
& \times \sum_{i=1}^{n} \int_{\mathbb{B}_{n}}\left|\frac{\partial K(z, w)}{\partial z_{i}}\right|^{2} d V(z) \\
& \leq C\|f\|_{2,1}^{2} \int_{D_{b}\left(\xi_{i}\right)} \frac{1}{\left(1-|w|^{2}\right)^{2 c}} K(w, w) d V(w) \\
& \leq C\|f\|_{2,1}^{2} \int_{D_{b}\left(\xi_{i}\right)} \frac{1}{\left(1-|w|^{2}\right)^{n+1+2 c}} d V(w) \\
& \leq C\|f\|_{2,1}^{2} \int_{0}^{1} \int_{\partial \mathbb{B}_{n}(r) \cap D_{b}\left(\xi_{i}\right)} \frac{1}{\left(1-r^{2}\right)^{n+2 c+1}} d S_{r} \\
& \leq C \lambda\|f\|_{2,1}^{2} \int_{0}^{1}\left(1-r^{2}\right)^{b-n-2 c-1} d r \leq C \lambda\|f\|_{2,1}^{2} \text {, }
\end{aligned}
$$

where $C$ is a constant and $\lambda$ is defined in (8); thus $\left\|T_{u_{i}}\right\| \leq$ $\sqrt{C \lambda}$. Set $T_{N}=\sum_{i=1}^{N}\left(1 / 2^{i}\right) T_{u_{i}}$; then $T_{N}$ is compact, and, for any $M, N \in \mathbb{N}$ and $f \in \mathscr{D}$, we find

$$
\left\|\sum_{i=N}^{M} \frac{1}{2^{i}} T_{u_{i}} f\right\|_{2,1} \leq \sqrt{C \lambda}\|f\|_{2,1} \sum_{i=N}^{M} \frac{1}{2^{i}}
$$

Equation (19) shows that $\left\|\sum_{i=N}^{M}\left(1 / 2^{i}\right) T_{u_{i}}\right\|_{2,1} \leq$ $\sqrt{C \lambda} \sum_{i=N}^{M}\left(1 / 2^{i}\right)$. Then $T_{N}=\sum_{i=1}^{N}\left(1 / 2^{i}\right) T_{u_{i}}$ converges to a compact operator $T=\sum_{i=1}^{\infty}\left(1 / 2^{i}\right) T_{u_{i}}$ in norm. It is obvious that $u_{i} \in L^{2}\left(\mathbb{B}_{n}\right)$ and $\left\|u_{i}\right\|_{2} \leq 1$; thus $u_{N}=\sum_{i=1}^{N} u_{i}$ converges to function $u=\sum_{i=1}^{\infty}\left(1 / 2^{i}\right) u_{i} \in L^{2}\left(\mathbb{B}_{n}\right)$ in norm. For any holomorphic polynomial $p$,

$$
\begin{aligned}
\left\|\left(T_{u}-T_{N}\right) p\right\|_{2,1} & =\left\|\left(\sum_{i=N+1}^{\infty} \frac{1}{2^{i}} T_{u_{i}}\right) p\right\|_{2,1} \\
& \leq\|p\|_{2,1} \sqrt{c \lambda} \sum_{i=N+1}^{\infty} \frac{1}{2^{i}} \longrightarrow 0,
\end{aligned}
$$

namely, $T=T_{u}$ which is the Toeplitz operator with symbol $u$. Hence, for any $\xi \in \partial \mathbb{B}_{n}$ and $r>0$,

$$
\operatorname{esssup}_{z \in \mathbb{B}_{n} \cap \mathbb{B}(\xi, r)}|u(z)|=\infty,
$$

where $\mathbb{B}(\xi, r)=\{z:|z-\xi|<r\}$, because of the density of set $\left\{\xi_{i}\right\}$ in unit surface.

Following the above theorem, we construct Schatten $p$ class Toeplitz operators for all $0<p<\infty$ whose symbols are also unbounded on any neighborhood of every point in unit surface.

Lemma 5 (See [5]). Let $f, g \in \mathscr{D}, d \mu=\varphi d A, \varphi \in L^{1}\left(\mathbb{B}_{n}, d A\right)$, Then

$$
\left\langle T_{\mu} f, g\right\rangle_{2,1}=\int_{\mathbb{B}_{n}} \varphi f \bar{g} d A
$$

Theorem 6. There is a function $u \in L^{1}\left(\mathbb{B}_{n}\right)$ which is unbounded on any neighborhood of each boundary point of $\mathbb{B}_{n}$ (i.e., for any $\xi \in \partial \mathbb{B}_{n}$ and $r>0$,

$$
\operatorname{esssup}_{z \in \mathbb{B}_{n} \cap \mathbb{B}(\xi, r)}|u(z)|=\infty,
$$

where $\mathbb{B}(\xi, r)=\{z:|z-\xi|<r\}) ; T_{u}$ is a Schatten p-class operator on Dirichlet-type space $\mathscr{D}$ for $0<p<\infty$.

Proof. For every multi-index $\alpha=\left(\alpha_{1}, \ldots, \alpha_{n}\right) \in \mathbb{N}^{+n}-$ $\{(0, \ldots, 0)\}$, we have $\left\|z^{\alpha}\right\|_{2,1}^{2}=n ! \alpha !|\alpha| /(|\alpha|+n-1)$ ! (see Rudin [13]). Thus $\left\{e_{\alpha}\right\}=\left\{z^{\alpha} /\left\|z^{\alpha}\right\|_{2,1}\right\}=\left\{\sqrt{|\alpha| n ! \alpha ! /(|\alpha|+n-1) !} z^{\alpha}\right\}$ forms the orthonormal base in $\mathscr{D}$. Choose a countable dense subset $\left\{\xi_{i}\right\}_{i=1}^{\infty}$ of $\partial \mathbb{B}_{n}$; let $U_{c}(z)=\left(1-|z|^{2}\right)^{-c}(c>0)$, and set

$$
u_{i}=\chi_{D_{b}\left(\xi_{i}, m\right)}(z) U_{c}(z),
$$

for any $m \in(0,1)$, where $b=2 c+((n+2) / p)(0<p \leq 1)$. Then

$$
\begin{aligned}
& \left|\left\langle T_{u_{i}} e_{\alpha}, e_{\alpha}\right\rangle_{2,1}\right| \\
& =\frac{(|\alpha|+n-1) !}{|\alpha| n ! \alpha !}\left|\int_{\mathbb{B}_{n}} u_{i}(w) w^{\alpha} \bar{w}^{\alpha} d V(w)\right| \\
& =\frac{(|\alpha|+n-1) !}{|\alpha| n ! \alpha !} \int_{D_{b}\left(\xi_{i}, m\right)}\left(1-|w|^{2}\right)^{-c} w^{\alpha} \bar{w}^{\alpha} d V(w) \\
& =C \frac{(|\alpha|+n-1) !}{|\alpha| n ! \alpha !} \int_{m}^{1} \int_{\partial \mathbb{B}_{n}(r) \cap D_{b}\left(\xi_{i}, m\right)}\left(1-r^{2}\right)^{-c} w^{\alpha} \cdot \bar{w}^{\alpha} d S_{r} d r \\
& \leq C \lambda \frac{(|\alpha|+n-1) !}{|\alpha| n ! \alpha !} \int_{m}^{1}\left(1-r^{2}\right)^{c+(n+2) / p} r^{2|\alpha|} d r
\end{aligned}
$$

where $C$ is a constant independent of $\alpha$. Integrating by parts, we find

$$
\int_{m}^{1}\left(1-r^{2}\right)^{c+(n+2) / p} r^{2|\alpha|} d r \leq \frac{d(n)}{(2|\alpha|+1)^{c+(n+2) / p+1}},
$$


where $d(n)$ is a constant that depends on $n$. Let

$$
T=\sum_{i=1}^{\infty} \frac{1}{2^{i}} T_{u_{i}}
$$

then $T$ is compact operator by Theorem 4 . Note that $T$ is positive operator; thus, for $0<p \leq 1$,

$$
\begin{aligned}
& \sum_{\alpha \in \mathbb{N}^{n}-\{(0, ; 0)\}}\left|\left\langle T e_{\alpha}, e_{\alpha}\right\rangle\right|^{p} \\
\leq & \sum_{\alpha \in \mathbb{N}^{n}-\{(0, ; 0)\}}\left(\sum_{i=1}^{\infty} \frac{1}{2^{i}} \frac{(|\alpha|+n-1) !}{|\alpha| n ! \alpha !} \frac{C \lambda d(n)}{(2|\alpha|+1)^{c+(n+2) / p+1}}\right)^{p} \\
\leq & \sum_{\alpha \in \mathbb{N}^{n}-\{(0, ; 0)\}} \sum_{i=1}^{\infty} \frac{1}{2^{i p}}\left(\frac{(|\alpha|+n-1) !}{|\alpha| n ! \alpha !}\right)^{p} \\
& \times\left(\frac{c \lambda d(n)}{\left.(2|\alpha|+1)^{c+(n+2) / p+1}\right)^{p}}\right. \\
\leq & \sum_{i=1}^{\infty} \frac{1}{2^{i p}} \sum_{\alpha \in \mathbb{N}^{n}-\{(0 ;, 0)\}}\left(\frac{(|\alpha|+n) !}{n ! \alpha !}\right)^{p} \\
& \times\left(\frac{c \lambda d(n)}{(2|\alpha|+1)^{c+(n+2) / p+1}}\right)^{p} \\
= & l \sum_{\alpha \in \mathbb{N}^{n}-\{(0,, 0)\}}\left(\frac{(|\alpha|+n) !}{n ! \alpha !}\right)^{p}\left(\frac{c \lambda d(n)}{(2|\alpha|+1)^{c+(n+2) / p+1}}\right)^{p}
\end{aligned}
$$

where $l=\sum_{i=1}^{\infty}\left(1 / 2^{i p}\right)$. Changing the order of summation, we have

$$
\begin{aligned}
\text { (28) } & =l \sum_{k=0}^{\infty}\left(\sum_{|\alpha|=k}\left(\frac{(|\alpha|+n) !}{n ! \alpha !}\right)^{p}\right)\left(\frac{c \lambda d(n)}{(2|\alpha|+1)^{c+(n+2) / p+1}}\right)^{p} \\
& =l \sum_{k=1}^{\infty}\left(\sum_{|\alpha|=k}\left(\frac{(k+n-1) !}{|\alpha| n ! \alpha !}\right)^{p}\right)\left(\frac{c \lambda d(n)}{(2 k+1)^{c+(n+2) / p+1}}\right)^{p} \\
& \leq l \sum_{k=1}^{\infty}\left(\sum_{|\alpha|=k} \frac{(k+n-1) !}{|\alpha| n ! \alpha !}\right)\left(\frac{c \lambda d(n)}{(2 k+1)^{c+(n+2) / p+1}}\right)^{p} .
\end{aligned}
$$

Note that, for any continuous function $f$ on $\mathbb{B}_{n}$, we have

$$
\begin{aligned}
& \int_{\mathbb{B}_{n}} f(z) d V(z) \\
& \quad=\int_{0}^{1} \int_{0}^{2 \pi}\left(\int_{\mathbb{B}_{n-1}\left(\sqrt{1-r^{2}}\right)} f\left(z^{\prime}, r e^{i \theta}\right) d V\left(z^{\prime}\right)\right) r d r d \theta,
\end{aligned}
$$

where $z^{\prime}=\left(z_{1}, \ldots, z_{n-1}\right), z_{n}=r e^{i \theta}, i=\sqrt{-1}$. Then, by induction, we see obviously that

$$
\sum_{|\alpha|=k} \frac{(k+n-1) !}{|\alpha| n ! \alpha !} \leq \sum_{|\alpha|=k} \frac{(k+n) !}{n ! \alpha !}=O\left((n+k)^{n}\right) .
$$

Further, there are constants $M_{1}, M_{2}>0$ such that

$$
\begin{aligned}
(29) & \leq M_{1} \sum_{k=0}^{\infty} \frac{(n+k)^{n}}{(2 k+1)^{(c+(n+2) / p+1) p}} \\
& \leq M_{2} \sum_{k=0}^{\infty} \frac{1}{(2 k+1)^{2}}<\infty .
\end{aligned}
$$

Namely, $T_{u}$ is a $S_{p}$-class operator for $0<p \leq 1$.

For $1<p<\infty$, let $b=n+2 c+2$; let $T$ be the operator defined in (27). By the above proof, we have

$$
\sum_{\alpha}\left|\left\langle T e_{\alpha}, e_{\alpha}\right\rangle_{2,1}\right|<\infty
$$

By the convergence in (33), we know that there exists a $N_{0}$ such that, for $|\alpha| \geq N_{0},\left|\left\langle T e_{\alpha}, e_{\alpha}\right\rangle_{2,1}\right|<1$; thus, we find $\left|\left\langle T e_{\alpha}, e_{\alpha}\right\rangle_{2,1}\right|^{p}<\left|\left\langle T e_{\alpha}, e_{\alpha}\right\rangle_{2,1}\right|$. Hence,

$$
\sum_{\alpha}\left|\left\langle T e_{\alpha}, e_{\alpha}\right\rangle_{2,1}\right|^{p}<\infty
$$

That is, $T_{u}$ is a $S_{p}$-class operator for $1<p<\infty$.

\section{Toeplitz Operators with Radial Symbols}

Is the product of two Toeplitz operators equal to a Toeplitz operator? In general, the answer is negative, but Brown and Halmos [14] showed that two bounded Toeplitz operators $T_{\varphi}$ and $T_{\psi}$ commute on the Hardy space if and only if (I) both $\varphi$ and $\psi$ are analytic, (II) both $\varphi$ and $\psi$ are analytic, or (III) one is a linear function of the other. For more details on the same question for Toeplitz operators on the Bergman spaces of one variable, see Cuckovic et al. $[15,16]$ and Louhichi et al. $[17,18]$.

For the case of the Bergman spaces of several variable, Zheng [19] studied commuting the Toeplitz operators with pluriharmonic symbols on the unit ball in $\mathbb{C}^{n}$. Recently, Quiroga-Barranco and Vasilevski $[20,21]$ gave the description of many (geometrically defined) classes of commuting Toeplitz operators on the unit ball. Zhou and Dong [22] discussed commuting Toeplitz operators with radial symbols on the unit ball.

In this section, we discuss the same questions for the Dirichlet-type space on the unit ball. The rest of this section is organized as follows. First, we introduce some basic properties of the Mellin transform and Mellin convolution which will be needed later. Second, we discuss when the product of two Toeplitz operators with radial symbols is a Toeplitz operator. Then, the zero-product problem for several Toeplitz operators with radial symbols on the Dirichlet-type space is investigated. Finally, the corresponding commuting problem of Toeplitz operators with quasihomogeneous symbols is studied.

3.1. Mellin Transform and Mellin Convolution. Mellin transform, the most useful tool we use later, is defined as follows. 
Definition 7. The Mellin transform $\widehat{u}$ of a function $u \in$ $L^{1}([0,1], r d r)$ is

$$
\widehat{u}(z)=\int_{0}^{1} u(s) s^{z-1} d s .
$$

It is known that $\widehat{u}$ is a bounded analytic function in the half plane $\{z: \operatorname{Re}(z)>2\}$. It is important and helpful to know that the Mellin transform is uniquely determined by its values on an arithmetic sequence of integers. In fact, we have the following classical theorem (see [23]).

Theorem 8. Let $f$ be a bounded analytic function on $\{z$ : $\operatorname{Re}(z)>0\}$ which vanishes at the pairwise distinct points $z_{1}, z_{2}, \ldots$, where

(1) $\inf \left\{\left|z_{n}\right|\right\}>0$,

(2) $\sum_{n \geq 1} \operatorname{Re}\left(1 / z_{n}\right)=\infty$.

Then $f$ vanishes identically on $\{z: \operatorname{Re}(z)>0\}$.

Remark 9. We will often use this theorem to show that if $u \in$ $L^{1}([0,1], r d r)$ and if there exists a sequence $\left\{n_{k}\right\}_{k \in \mathbb{Z}^{+}} \subset \mathbb{N}$ such that

$$
\widehat{u}\left(n_{k}\right)=0, \quad \sum_{k=1}^{\infty} \frac{1}{n_{k}}=\infty,
$$

then $\widehat{u}=0$ for all $z \in\{z: \operatorname{Re}(z)>2\}$ and so $u=0$.

When considering the product of two Toeplitz operators, we need a known fact about the Mellin convolution of their symbols. If $f$ and $g$ are defined on $[0,1)$, then their Mellin convolution is defined by

$$
\left(f *_{M} g\right)(r)=\int_{r}^{1} f\left(\frac{r}{t}\right) g(t) \frac{d t}{t}, \quad 0 \leq r<1 .
$$

The Mellin convolution theorem states that

$$
\left(\widehat{f *_{M} g}\right)(r)=\widehat{f}(r) \widehat{g}(r)
$$

and that if $f$ and $g$ are in $L^{1}([0,1], r d r)$, then so is $\left(\overline{f *_{M} g}\right)(r)$.

3.2. Products of Toeplitz Operators with Radial Symbols. For convenience, we use $\alpha-\beta$ to denote

$$
\left(\alpha_{1}-\beta_{1}, \ldots, \alpha_{n}-\beta_{n}\right) \text {. }
$$

The notations $\alpha \geq \beta$ and $\alpha \perp \beta$ mean, respectively,

$$
\begin{gathered}
\alpha_{i} \geq \beta_{i}, \quad i=1, \ldots, n, \\
\sum_{i=1}^{n} \alpha_{i} \beta_{i}=0 .
\end{gathered}
$$

It is trivial that when $\alpha \geq \beta$,

$$
|\alpha-\beta|=|\alpha|-|\beta| \text {. }
$$

A function $u$ on $B_{n}$ is called the radial function, if $u(z)$ depends only on $|z|$. It is obvious that $u$ is radial if and only if $u(U z)=u(z)$ for any unitary transform $U$ of $\mathbb{C}^{n}$. Then, for each radial function $u$, we define the function $\tilde{u}$ on $[0$, 1) by $\tilde{u}(r)=u(r \mathbf{e})$ where $\mathbf{e}$ is a unit vector in $\mathbb{C}^{n}$. It is trivial that $\tilde{u}$ is well defined. In the following, we will often identify an integrable radial function $u$ on the unit ball with the corresponding function $\tilde{u}$ defined on the interval $[0,1)$.

In the following, some basic results concerning Toeplitz operators with radial symbols on the Dirichlet-type space of the unit ball are obtained.

Theorem 10 (see Lu and Sun [5]). Let $u$ be a radial function and $b_{\alpha}=(2 /|\alpha|) \int_{0}^{1} r^{2 n+2|\alpha|-1} u(r) d r$. Then,

(1) $T_{u}$ is bounded if and only if $\sup _{\alpha}\left|b_{\alpha}\right|<\infty$;

(2) $T_{u}$ is compact if and only if $b_{\alpha} \rightarrow 0(|\alpha| \rightarrow \infty)$.

Theorem 11. Let $u \in L^{1}\left(\mathbb{B}_{n}\right)$ be a radial function in which $T_{u}$ is bounded on $\mathscr{D}$; then, for any $\alpha \in \mathbb{N}^{n}-\{(0, \ldots, 0)\}$,

$$
\left(T_{u} z^{\alpha}\right)(w)=\frac{2}{|\alpha|} \widehat{u}(2 n+2|\alpha|) w^{\alpha}
$$

Proof. By the definition of Toeplitz operator, we have

$$
\begin{aligned}
& \left(T_{u} z^{\alpha}\right)(w) \\
& =\int_{\mathbb{B}_{n}} u(z) z^{\alpha} \sum_{\beta \in \mathbb{N}^{n}-\{(0, \ldots, 0)\}} \frac{(|\beta|+n-1) !}{|\beta| n ! \alpha !} \bar{z}^{\beta} w^{\beta} d A \\
& =\sum_{\beta \in \mathbb{N}^{n}-\{(0, \ldots, 0)\}} w^{\beta} \int_{\mathbb{B}_{n}} u(z) z^{\alpha} \frac{(|\beta|+n-1) !}{|\beta| n ! \beta !} \bar{z}^{\beta} d A .
\end{aligned}
$$

From [13], we know the unique nonzero item in (43) is

$$
\left(T_{u} z^{\alpha}\right)(w)=w^{\alpha} \int_{\mathbb{B}_{n}} u(z) z^{\alpha} \frac{(|\alpha|+n-1) !}{|\alpha| n ! \alpha !} \bar{z}^{\alpha} d A
$$

A direct computation shows that

$$
\begin{aligned}
& \left(T_{u} z^{\alpha}\right)(w) \\
& =2 n \frac{(|\alpha|+n-1) !}{|\alpha| n ! \alpha !} w^{\alpha} \int_{0}^{1} r^{2 n-1} d r \int_{\partial \mathbb{B}_{n}} u(r)(r \xi)^{\alpha} \overline{(r \xi)}^{\alpha} d S \\
& =\frac{2}{|\alpha|} w^{\alpha} \int_{0}^{1} u(r) r^{2 n+2|\alpha|-1} d r=\frac{2}{|\alpha|} \widehat{u}(2 n+2|\alpha|) w^{\alpha} .
\end{aligned}
$$

Theorem 11 shows that the Toeplitz operator with a radial symbol on the Dirichlet-type space of the unit ball acts in a very simple way. On the other hand, the Toeplitz operators of Theorem 11 must be Toeplitz operators with radial symbols.

Theorem 12. Let $u \in L^{1}\left(\mathbb{B}_{n}\right)$. For each multi-index $\alpha$, if there exists $\lambda_{|\alpha|} \in \mathbb{C}$ which depends only on $|\alpha|$ such that $T_{u} z^{\alpha}=$ $\lambda_{|\alpha|} z^{\alpha}$, then $u$ is a radial function. 
Proof. Suppose $T_{u} z^{\alpha}=\lambda_{|\alpha|} z^{\alpha}$; then, for any unitary transform $U$ of $\mathbb{C}^{n}$ with

$$
U^{-1}=\left(a_{1}, \ldots, a_{n}\right)^{T}=\left(a_{i j}\right)_{1 \leq i, j \leq n}
$$

there holds

$$
\begin{aligned}
& T_{u}\left(U^{-1} z\right)^{\alpha} \\
& =T_{u}\left[\left(a_{11} z_{1}+\cdots+a_{1 n} z_{n}\right)^{\alpha_{1}} \cdots\left(a_{n 1} z_{1}+\cdots+a_{n n} z_{n}\right)^{\alpha_{n}}\right] \\
& =T_{u}\left[\left(\sum_{\left|\beta^{1}\right|=\alpha_{1}} \frac{\alpha^{1} !}{\beta^{1} !}\left(a_{11} z_{1}\right)^{\beta_{1}^{1}} \cdots\left(a_{1 n} z_{n}\right)^{\beta_{n}^{1}}\right)\right. \\
& \left.\times \cdots \times\left(\sum_{\left|\beta^{n}\right|=\alpha_{n}} \frac{\alpha_{n} !}{\beta^{n !}}\left(a_{n 1} z_{1}\right)^{\beta_{1}^{n}} \cdots\left(a_{n n} z_{n}\right)^{\beta_{n}^{n}}\right)\right] \\
& =\sum_{\left|\beta^{1}\right|=\alpha_{1}} \cdots \sum_{\left|\beta^{n}\right|=\alpha_{n}} \frac{\alpha_{1} !}{\beta^{1} !} \cdots \frac{\alpha_{n} !}{\beta^{n !}}\left(a_{1}\right)^{\beta^{1}} \cdots\left(a_{n}\right)^{\beta^{n}} \\
& \times T_{u}\left(z^{\beta^{1}} \cdots z^{\beta^{n}}\right) \\
& =\lambda_{|\alpha|} \sum_{\left|\beta^{1}\right|=\alpha_{1}} \cdots \sum_{\left|\beta^{n}\right|=\alpha_{n}} \frac{\alpha_{1} !}{\beta^{1} !} \cdots \frac{\alpha_{n} !}{\beta^{n !}}\left(a_{1}\right)^{\beta^{1}} \cdots\left(a_{n}\right)^{\beta^{n}} \\
& \times\left(z^{\beta^{1}} \cdots z^{\beta^{n}}\right) \\
& =\lambda_{|\alpha|}\left(U^{-1} z\right)^{\alpha}
\end{aligned}
$$

where $\beta^{1}, \ldots, \beta^{n}$ are multi-indexes. By the definition of Toeplitz operator, note that $\langle z, w\rangle=\sum_{i=1}^{n} z_{i} \bar{w}_{i}$; we have

$$
\begin{aligned}
& \left(T_{u_{0} U^{\prime}} z^{\alpha}\right)(w) \\
& =\int_{\mathbb{B}_{n}} u(U z) z^{\alpha} K(w, z) d A(z) \\
& =\int_{\mathbb{B}_{n}} u(U z) z^{\alpha} \sum_{\beta \in \mathbb{N}^{n}-\{(0, \ldots, 0)\}} \frac{(|\beta|+n-1) !}{|\beta| n ! \beta !} w^{\beta} \bar{z}^{\beta} d A(z) \\
& =\int_{\mathbb{B}_{n}} u(U z) z^{\alpha} \sum_{k=1} \frac{(n+k-1) !}{k n ! k !} \sum_{k \mid=k} \frac{k !}{\beta !} w^{\beta} \bar{z}^{\beta} d A(z) \\
& =\int_{\mathbb{B}_{n}} u(U z) z^{\alpha} \sum_{k=1} \frac{(n+k-1) !}{k n ! k !}\langle w, z\rangle^{k} d A(z) \\
& =\int_{\mathbb{B}_{n}} u(z)\left(U^{-1} z\right)^{\alpha} \sum_{k=1} \frac{(n+k-1) !}{k n ! k !}\left\langle w, U^{-1} z\right\rangle^{k} d A(z) \\
& =\int_{\mathbb{B}_{n}} u(z)\left(U^{-1} z\right)^{\alpha} \sum_{k=1} \frac{(n+k-1) !}{k n ! k !}\langle U w, z\rangle^{k} d A(z) \\
& \left.=\left(T_{u}(U)^{-1} z\right)^{\alpha}\right)(U w)=\lambda\left(w^{\alpha}=\left(T_{u} z^{\alpha}\right)(w),\right.
\end{aligned}
$$

which shows that $T_{u \circ U}=T_{u}$. Thus, $u \circ U=u$ and $u$ is a radial function.

Corollary 13. Let $u_{1}$ and $u_{2}$ be two radial functions on $\mathbb{B}_{n}$ such that $T_{u_{1}}, T_{u_{2}}$ are bounded. If $T_{u_{1}} T_{u_{2}}=T_{v}$, then $v$ is a radial function and $T_{v}$ is a bounded operator.

Proof. Using (42) to calculate $T_{u_{1}} T_{u_{2}} z^{\alpha}$, we obtain

$$
T_{u_{1}} T_{u_{2}} z^{\alpha}=\frac{4}{|\alpha|^{2}} \widehat{u_{1}}(2 n+2|\alpha|) \widehat{u_{2}}(2 n+2|\alpha|) z^{\alpha} .
$$

From Theorem 12, $v$ is a radial function. Moreover, $T_{v}$ is obviously a bounded operator.

Grudsky et al. [24] gave a particular answer to this question considering the Toeplitz operators with radial symbols. In [22], Zhou and Dong discussed the same question about the Toeplitz operators with radial symbols; they gave a different way to characterize when the product of two Toeplitz operators is equal to a Toeplitz operator. Let $u_{1}$ and $u_{2}$ be two radial functions on $\mathbb{B}_{n}$ which induce bounded Toeplitz operators, and let further

$$
A_{1}(t)=u_{1}\left(\sqrt{e^{-t}}\right), \quad A_{2}(t)=u_{2}\left(\sqrt{e^{-t}}\right) .
$$
form)

The formal construction (inverse Fourier-Laplace trans-

$$
\mathscr{F}^{-1} A(z)=\frac{1}{\sqrt{2 \pi}} \int_{\mathbb{R}} A(t) e^{i z t} d t, \quad z \in \Pi \cup \mathbb{R},
$$

defines a holomorphic function in the upper half plane $\Pi \subset$ $\mathbb{C}$ which coincides on the real axis with the inverse Fourier transform $\mathscr{F}^{-1} A_{1}(\zeta)$ of the function $A_{1}(t)$. Theorem 3.7 of [24] shows that if the function

$$
\sqrt{2 \pi}(n-i \zeta) \mathscr{F}^{-1} A_{1}(\zeta) \mathscr{F}^{-1} A_{2}(\zeta), \quad \zeta \in \mathbb{R}
$$

belongs to Wiener ring $W_{0}$ of the inverse Fourier transforms of summable functions, then there exists a Toeplitz operator with the radial symbol $v$ such that $T_{u_{1}} T_{u_{2}}=T_{v}$. The following theorem will give the condition for the product of two Toeplitz operators with radial symbols to be a Toeplitz operator on Dirichlet-type space.

Theorem 14. Let $u_{1}$ and $u_{2}$ be two radial functions on $\mathbb{B}_{n}$ which induce bounded Toeplitz operators. Then, $T_{u_{1}} T_{u_{2}}$ is equal to the Toeplitz operator $T_{v}$ if and only if

$$
v=\left(4|z|^{-2 n}\right) *_{M} u_{1} *_{M} u_{2} .
$$

Proof. For any $\alpha$, it follows from (42) and (49) that

$$
T_{u_{1}} T_{u_{2}} z^{\alpha}=T_{v} z^{\alpha}
$$

if and only if

$$
\begin{aligned}
\widehat{v}(2 n+2|\alpha|) & =\frac{2}{|\alpha|} \widehat{u_{1}}(2 n+2|\alpha|) \widehat{u_{2}}(2 n+2|\alpha|) \\
& =\frac{2}{|\alpha|} \widehat{u_{1} * M^{u_{2}}}(2 n+2|\alpha|) .
\end{aligned}
$$


A direct computation gives

$$
\begin{aligned}
\widehat{v}(2 n+2|\alpha|) & =\widehat{u_{1} * M^{u_{2}}}(2 n+2|\alpha|) \int_{0}^{1} 4 s^{-2 n} s^{2 n+2|\alpha|-1} d s \\
& =\widehat{4 r^{-2 n}}(2 n+2|\alpha|) \widehat{u_{1} *_{M} u_{2}}(2 n+2|\alpha|) \\
& =\left(\left(4 r^{-2 n} \widehat{*_{M} u_{1}} *{ }_{M} u_{2}\right)(2 n+2|\alpha|),\right.
\end{aligned}
$$

where $\widehat{4 r^{-2 n}}(z)$ is well defined if $\operatorname{Re}(z)>2 n$. By Remark 9, (56) is equivalent to (53).

For some products of Toeplitz operators, the following fun result is obtained.

Corollary 15. Let $l$ and $m$ be two real numbers greater than or equal to $-2 n$. Then,

$$
\begin{aligned}
& T_{|z|^{l}} T_{|z|^{m}} \\
& =\left\{\begin{array}{c}
\frac{4}{(2 n+m)(2 n+l)} T_{|z|^{-2 n}}+\frac{4}{(2 n+l)(l-m)} T_{|z|^{l}} \\
-\frac{4}{(2 n+m)(l-m)} T_{|z|^{m}} \\
\text { if } l \neq m, \\
\frac{4}{2 n+l} T_{|z|^{l} \ln |z|}+\frac{4}{(2 n+l)^{2}} T_{|z|^{-2 n}}-\frac{4}{(2 n+l)^{2}} T_{|z|^{l}} \\
\text { if } l=m .
\end{array}\right.
\end{aligned}
$$

Proof. By Theorem 14, $T_{|z|} T_{|z|^{m}}$ is equal to the Toeplitz operator $T_{v}$ if and only if

$$
v=\left(4|z|^{-2 n}\right) *_{M} u_{1} *_{M} u_{2}
$$

A direct calculation shows that

$$
v=\left\{\begin{array}{c}
\frac{4}{(2 n+m)(2 n+l)}|z|^{-2 n}+\frac{4}{(2 n+l)(l-m)}|z|^{l} \\
-\frac{4}{(2 n+m)(l-m)}|z|^{m} \\
\text { if } l \neq m, \\
\frac{4}{2 n+l}|z|^{l} \ln |z|+\frac{4}{(2 n+l)^{2}}|z|^{-2 n}-\frac{4}{(2 n+l)^{2}}|z|^{l} \\
\text { if } l=m .
\end{array}\right.
$$

The desired result follows from (59).

Axler and Cuckovic [16] and Choe and Koo [25] study, respectively, the zero-product problem for two Toeplitz operators with harmonic symbols on unit disk and on the Bergman spaces of the unit ball. In [22], Zhou and Dong discuss the same question about the Toeplitz operators with radial symbols. In the following theorem, we will solve the zero-product problem for several Toeplitz operators with radial symbols acting on the Dirichlet-type space of the unit ball.
Theorem 16. Suppose that $u_{1}, \ldots, u_{N}$ are radial functions on $\mathbb{B}_{n}$ that induce bounded Toeplitz operators on Dirichlet-type space. If $T_{u_{1}} \cdots T_{u_{N}}=0$, then $u_{i}=0$ for some $i$.

Proof. If $T_{u_{1}} \cdots T_{u_{N}}=0$, then, for any multi-index $\alpha$, by Theorem 11, there holds

$$
\widehat{u_{1}}(2 n+2|\alpha|) \cdots \widehat{u_{N}}(2 n+2|\alpha|)=0 .
$$

Set

$$
E_{i}=\left\{|\alpha| \in \mathbb{N}: u_{i}(2 n+2|\alpha|)=0\right\}
$$

Since $\cup_{i=1}^{n} E_{i}=\mathbb{N}$, there exists an $E_{i}$ such that

$$
\sum_{|\alpha| \in E_{i}} \frac{1}{2 n+2|\alpha|}=\infty
$$

then, by Remark 9, $u_{i}=0$.

By Theorem 16, we can show that the only idempotent Toeplitz operators with radial symbols are 0 and $I$.

Corollary 17. If $u$ is a radial function on $\mathbb{B}_{n}$, then $T_{u}^{2}=T_{u}$ if and only if either $u=0$ or $u=1$.

Proof. If $T_{u}^{2}=T_{u}$, then $T_{u} T_{u-1}=0$, and, by Theorem $16, u=0$ or $u=1$. The converse implication is trivial.

3.3. Commuting Toeplitz Operators with Quasihomogeneous Symbols on Dirichlet-Type Space. In this subsection, commuting Toeplitz operators with bounded quasihomogeneous symbols on the Dirichlet-type space of the unit ball are discussed. The definition of the quasihomogeneous function on the unit disk has been given in $[17,26]$, and the definition on the unit ball has been given in [22].

Definition 18. Let $\lambda \in \mathbb{Z}^{n}$ and $u \in L^{1}\left(\mathbb{B}_{n}, d A\right)$. $u$ is called a quasihomogeneous function of quasihomogeneous degree $\lambda$ if $u$ is of the form $\xi^{\lambda} \varphi$, where $u$ is a radial function; that is,

$$
u(r \xi)=\xi^{k} \varphi(r)
$$

for any $\xi$ in the unit sphere $S$ and $r \in[0,1)$.

Remark 19. It is obvious that any $\lambda \in \mathbb{Z}^{n}$ can be uniquely written as $\beta-\gamma$, where $\beta$ and $\gamma$ are two multi-indexes such that $\beta \perp \gamma$. Thus, in this paper, we always define the function

$$
\xi^{\lambda}=\xi^{\beta} \bar{\xi}^{\gamma}, \quad \xi \in S_{n}
$$

for any $\lambda \in \mathbb{Z}^{n}$.

In the following lemma, a result which we will use often is given. 
Lemma 20. Let $\beta, \gamma$ be two multi-indexes and let $u$ be a bounded radial function on $\mathbb{B}_{n}$. Then, for any multi-index $\alpha$,

$$
\begin{aligned}
& T_{\xi^{\beta} u}\left(z^{\alpha}\right)=\frac{2}{|\alpha|+|\beta|} \widehat{u}(2 n+2|\alpha|+|\beta|) z^{\alpha+\beta} ; \\
& T_{\bar{\xi}^{\gamma} u}\left(z^{\alpha}\right)=\left\{\begin{array}{cc}
0, & \text { if } \alpha \nsucceq \gamma, \\
\frac{2 \alpha !(|\alpha|-|\gamma|+n-1) !}{(|\alpha|+n-1) !(|\alpha|-|\gamma|)(\alpha-\gamma) !} \\
\times \widehat{u}(2 n+2|\alpha|-|\gamma|) z^{\alpha-\gamma}, \\
\text { if } \alpha \geq \gamma ;
\end{array}\right. \\
& T_{\xi \beta \bar{\xi}^{\gamma} u}\left(z^{\alpha}\right) \\
& = \begin{cases}0, & \text { if } \alpha+\beta \nsucceq \gamma, \\
\frac{2(\alpha+\beta) !(|\alpha|+|\beta|-|\gamma|+n-1) !}{(|\alpha|+|\beta|+n-1) !(|\alpha|+|\beta|-|\gamma|)(\alpha+\beta-\gamma) !} \\
\times \widehat{u}(2 n+2|\alpha|+|\beta|-|\gamma|) z^{\alpha+\beta-\gamma}, \\
\text { if } \alpha+\beta \succeq \gamma .\end{cases}
\end{aligned}
$$

Proof. We just need to prove (67). For any $\alpha \in \mathbb{N}^{n}-$ $\{(0, \ldots, 0)\}$,

$$
\begin{aligned}
& \left(T_{\xi^{\beta} \bar{\xi}^{\gamma} u^{\prime}} z^{\alpha}\right)(w) \\
& =\int_{\mathbb{B}_{n}} \xi^{\beta} \bar{\xi}^{\gamma} u(|z|) z^{\alpha} \\
& \times \sum_{\zeta \in \mathbb{N}^{n}-\{(0, \ldots, 0)\}} \frac{(|\zeta|+n-1) !}{|\zeta| n ! \zeta !} w^{\zeta} \bar{z}^{\zeta} d A \\
& =\sum_{\zeta \in \mathbb{N}^{n}-\{(0, \ldots, 0)\}} \frac{(|\zeta|+n-1) !}{|\zeta| n ! \zeta !} w^{\zeta} 2 n \\
& \times \int_{0}^{1} r^{2 n-1+|\alpha|+|\zeta|} u(r) d r \int_{\partial \mathbb{B}_{n}} \xi^{\beta+\alpha} \bar{\xi}^{\gamma+\zeta} d S \\
& =\left\{\begin{array}{l}
0, \quad \text { if } \alpha+\beta \nsucceq \gamma, \\
\sum_{\zeta \in \mathbb{N}^{n}-\{(0, \ldots, 0)\}} \frac{(|\zeta|+n-1) !}{|\zeta| n ! \zeta !} w^{\zeta} 2 n \widehat{u}(2 n+|\alpha|+|\zeta|) \\
\times \int_{\partial \mathbb{B}_{n}} \xi^{\beta+\alpha} \bar{\xi}^{\gamma+\zeta} d S, \\
\text { if } \alpha+\beta \succeq \gamma,
\end{array}\right. \\
& =\left\{\begin{aligned}
0, & \text { if } \alpha+\beta \nsucceq \gamma, \\
2 n \widehat{u}(2 n+2|\alpha|+|\beta|-|\gamma|) \int_{\partial \mathbb{B}_{n}} \xi^{\beta+\alpha} \bar{\xi}^{\beta+\alpha} d S & \\
& \times \frac{(|\alpha|+|\beta|-|\gamma|+n-1) !}{(|\alpha|+|\beta|-|\gamma|) n !(\alpha+\beta-\gamma) !} w^{\alpha+\beta-\gamma}, \\
& \text { if } \alpha+\beta \succeq \gamma .
\end{aligned}\right.
\end{aligned}
$$

By calculation, we get the desired results.

Now, we can discuss the commuting problem of Toeplitz operators with quasihomogeneous symbols on Dirichlet-type space.
Theorem 21. Let $\beta, \gamma$ be two multi-indexes and let $u$ and $v$ be two bounded radial functions on $\mathbb{B}_{n}$. If $u$ is not equal to 0 , then

$$
T_{u} T_{\xi^{\beta} \bar{\xi}^{\gamma} v}=T_{\xi^{\beta} \bar{\xi}^{\gamma} v} T_{u}
$$

if and only if either $|\beta|=|\gamma|$ or $v=0$.

Proof. If (69) holds, then for any multi-index $\alpha$ such that $\alpha+$ $\beta \geq \gamma$; by Theorem 11 and Lemma 20, we have

$$
\begin{aligned}
& \frac{1}{|\alpha|+|\beta|-|\gamma|} \widehat{u}(2 n+2|\alpha|+2|\beta|-2|\gamma|) \\
& \quad \times \widehat{v}(2 n+2|\alpha|+|\beta|-|\gamma|) \\
& =\frac{1}{|\alpha|} \widehat{u}(2 n+2|\alpha|) \widehat{v}(2 n+2|\alpha|+|\beta|-|\gamma|) .
\end{aligned}
$$

Assume that $|\beta| \neq|\gamma|$; without loss of generality, we can also assume that $|\beta|>|\gamma|$, for otherwise we could take the adjoins. Set

$$
E=\{|\alpha| \in \mathbb{N}: \widehat{v}(2 n+2|\alpha|+|\beta|-|\gamma|)=0\} .
$$

It will show later that $\sum_{\alpha \in E}(1 /|\alpha|)=\infty$, which implies that $v=0$ by Remark 9 .

If $\sum_{\alpha \in E}(1 /|\alpha|)<\infty$, we will induce a contradiction. Let

$$
E^{\prime}=E^{c} \cap\left\{|\alpha| \in \mathbb{N}: \alpha \in \mathbb{Z}^{+}, \alpha+\beta \succeq \gamma\right\},
$$

where $E^{c}$ is the complement of $E$ in $\mathbb{N}$; then

$$
\sum_{\alpha \in E^{\prime}} \frac{1}{|\alpha|}=\infty
$$

On the other hand, for any $|\alpha| \in E^{c}$, (70) gives

$$
\begin{aligned}
& \frac{1}{|\alpha|+|\beta|-|\gamma|} \widehat{u}(2 n+2|\alpha|+2|\beta|-2|\gamma|) \\
& =\frac{1}{|\alpha|} \widehat{u}(2 n+2|\alpha|) .
\end{aligned}
$$

Denote

$$
F(z)=\widehat{u}(z+2 n+2|\beta|-2|\gamma|)-\frac{z+2|\beta|-2|\gamma|}{z} \widehat{u}(z+2 n)
$$

then $F$ is analytic and bounded on $\{z: \operatorname{Re}(z)>0\}$ since $v$ is bounded. Moreover, (74) implies that

$$
F(2|\alpha|)=0, \quad \forall|\alpha| \in E^{\prime} .
$$

According to Theorem $8, F$ must be zero; thus,

$$
\begin{aligned}
& \frac{1}{z+2|\beta|-2|\gamma|} \widehat{u}(z+2 n+2|\beta|-2|\gamma|) \\
& =\frac{1}{z} \widehat{u}(z+2 n+2|\alpha|) \quad z \in\{z: \operatorname{Re}(z)>0\} .
\end{aligned}
$$


For any integer $n_{0}$ greater than $2 n$, the above equation gives that

$$
\begin{gathered}
\frac{1}{n_{0}-2 n+2 m(|\beta|-|\gamma|)} \widehat{u}\left(n_{0}+2 m(|\beta|-|\gamma|)\right) \\
=\frac{1}{n_{0}-2 n} \widehat{u}\left(n_{0}\right) \quad \forall m \in \mathbb{N} .
\end{gathered}
$$

If we denote $\left(1 /\left(n_{0}-2 n\right)\right) \widehat{u}\left(n_{0}\right)$ by the constant $C$, we obtain

$$
\begin{gathered}
\frac{1}{\left(n_{0}-2 n+2 m(|\beta|-|\gamma|)\right)} \widehat{u}\left(n_{0}+2 m(|\beta|-|\gamma|)\right) \\
=|z|^{-2 n} * u=C .
\end{gathered}
$$

Multiply two sides of (79) by $1 /\left(n_{0}+2 m(|\beta|-|\gamma|)\right)$, which leads to

$$
\begin{gathered}
\left(\llbracket * \widehat{|z|^{-2 n}} * u\right)\left(n_{0}+2 m(|\beta|-|\gamma|)\right) \\
=\widehat{C \square}\left(n_{0}+2 m(|\beta|-|\gamma|)\right),
\end{gathered}
$$

where $\llbracket$ denotes the constant function with value one, for any $m \in \mathbb{N}$, by Remark 9 again, and, then, clearly, $\left(\llbracket *|z|^{-2 n} * u\right)$ is constant.

By calculation, we know that

$$
\square *|z|^{-2 n} * u(r)=\int_{r}^{1} \frac{1}{2 n}\left(\frac{t^{2 n}}{r^{2 n}}-1\right) \frac{u(t)}{t} d t=C ;
$$

then, derive (81) with respect to variable $r$; we get

$$
\begin{gathered}
\frac{1}{-2 n} r^{-2 n-1} \int_{r}^{1} t^{2 n-1} u(t) d t-r^{-2 n} r^{2 n-1} u(r)+u(r) r^{-1} \\
=\frac{1}{2 n} r^{-2 n-1} \int_{r}^{1} t^{2 n-1} u(t) d t=0
\end{gathered}
$$

and, then, clearly, $u$ is 0 , which is a contradiction. Thus, we conclude that either $|\beta|=|\gamma|$ or $v=0$.

Conversely, if $|\beta|=|\gamma|$ or $v=0$, then we can easily show that (70) holds, and, consequently,

$$
T_{u} T_{\xi^{\beta} \bar{\xi}^{v} v}=T_{\xi^{\beta} \bar{\xi}^{v}{ }_{v}} T_{u}
$$

for each multi-index $\alpha$, which implies $T_{\xi^{\beta} \bar{\xi}^{\gamma}{ }_{v}}$ and $T_{u}$ commute.

It was shown in [27] that a Toeplitz operator with a radial symbol on the Bergman spaces of the unit disk $D$ may only commute with another such operator with a radial symbol, but it is not true in higher dimensions by the theorem above. It is known that every function $f \in L^{2}(D, d A)$ has the decomposition

$$
f\left(r e^{i k \theta}\right)=\sum_{k=-\infty}^{\infty} e^{i k \theta} f_{k}(r)
$$

where $f_{k}(r)$ are square integrables in $[0,1]$ with respect to the measure $r d r$. More details can be found in [27]. Similarly, let

$$
R=\left\{u: \mathbb{B}_{n} \longrightarrow \mathbb{C} \text { radial }: \int_{0}^{1} r^{2 n-1}|u|^{2} d r<\infty\right\}
$$

Denote $R_{k}=\xi^{k} R$ for $k \in Z^{n}$. Each $R_{k}$ is a subspace of $L^{2}\left(\mathbb{B}_{n}, d A\right)$ since, for $f \in R_{k}, f(r \xi)=\xi^{k} u(r)$ and

$$
\begin{aligned}
& \int_{\mathbb{B}_{n}}|f(z)|^{2} d A(z) \\
& \quad=2 n \int_{0}^{1} r^{2 n+1}|u(r)|^{2} d r \int_{\partial \mathbb{B}_{n}}\left|\xi^{k}\right|^{2} d S(\xi)<\infty .
\end{aligned}
$$

It is also clear that $R_{k} \perp R_{l}$ if $k \neq l$. Obviously, there exist many square-integrable functions $f(z)$ on $\mathbb{B}_{n}$ which do not have the decomposition $f(r \xi)=\sum_{k \in Z^{n}} \xi^{k} f_{k}(r)$, but we still might study a function of that form. In the one-dimensional case, the function $f(r \xi)=\sum_{k \in Z^{n}} \xi^{k} f_{k}(r)$ is exactly the same as (84). Moreover, if

$$
f(r \xi)=\sum_{k \in \mathbb{Z}^{n}} \xi^{k} f_{k}(r) \in L^{\infty}\left(\mathbb{B}_{n}, d A\right),
$$

then, for each $k \in \mathbb{Z}^{n}$

$$
\left|f_{k}(r)\right|=\frac{1}{\left\|\xi^{k}\right\|^{2}}\left|\int_{\partial \mathbb{B}_{n}} f_{k}(r) \bar{\xi}^{k} d S(\xi)\right| \leq \sup _{z \in \mathbb{B}_{n}}|f(z)|,
$$

and so the functions $f_{k}$ are bounded on $\mathbb{B}_{n}$.

Lemma 22. Let $\beta \in \mathbb{N}^{n}$ be a multi-index and let $u$ be $a$ bounded radial function on $\mathbb{B}_{n}$. If

$$
f(r \xi)=\sum_{k \in \mathbb{Z}^{n}} \xi^{k} f_{k}(r) \in L^{\infty}\left(\mathbb{B}_{n}, d A\right),
$$

then

$$
T_{f} T_{\xi^{\beta} u}=T_{\xi^{\beta} u} T_{f} \Longleftrightarrow T_{\xi^{k} f_{k}} T_{\xi^{\beta} u}=T_{\xi^{\beta} u} T_{\xi^{k} f_{k}}, \quad \forall k \in \mathbb{Z}^{n} .
$$

Proof. Suppose that $f(r \xi)=\sum_{k \in \mathbb{Z}^{n}} \xi^{k} f_{k}(r) \in L^{\infty}\left(\mathbb{B}_{n}, d A\right)$; then, for any multi-index $\alpha$, a direct calculation by Lemma 20 gives that

$$
\begin{aligned}
& T_{f} T_{\xi^{\beta} u} z^{\alpha}=\sum_{k+\alpha+\beta \succeq 0} T_{\xi^{k} f} T_{\xi^{\beta} u} z^{\alpha}, \\
& T_{\xi^{\beta} u} T_{f} z^{\alpha}=\sum_{k+\alpha+\beta \succeq 0} T_{\xi^{\beta} u} T_{\xi^{k} f} z^{\alpha} .
\end{aligned}
$$

If $T_{f}$ and $T_{\xi^{\beta} u}$ commute, the equality of the above two series implies that

$$
T_{\xi^{k} f} T_{\xi^{\beta} u} z^{\alpha}= \begin{cases}0, & \text { if } k+\alpha+\beta \nsucceq 0, k+\alpha \nsucceq 0, \\ T_{\xi^{\beta} u} T_{\xi^{k}} z^{\alpha}, & \text { if } k+\alpha \succeq 0 .\end{cases}
$$


Recall that $T_{\xi^{\beta} u} T_{\xi^{k}} z^{\alpha}$ is equal to zero if $k+\alpha \nsucceq 0$, then, for each multi-index $\alpha$ and for all $k \in \mathbb{Z}^{n}$,

$$
T_{\xi^{k} f} T_{\xi^{\beta} u} z^{\alpha}=T_{\xi^{\beta} u} T_{\xi^{k} f} z^{\alpha}
$$

Thus, $T_{\xi^{k} f} T_{\xi^{\beta} u}=T_{\xi^{\beta} u} T_{\xi^{k}}, \forall k \in \mathbb{Z}^{n}$. The converse implication is clear.

Corollary 23. Let $u$ be a nonzero bounded radial function on $\mathbb{B}_{n}$. If

$$
f(r \xi)=\sum_{k \in \mathbb{Z}^{n}} \xi^{k} f_{k}(r) \in L^{\infty}\left(\mathbb{B}_{n}, d V\right)
$$

then $T_{u} T_{f}=T_{f} T_{u}$ if and only if $f\left(e^{i \theta} z\right)=f(z)$ for almost all $\theta \in \mathbb{R}$ and $z \in \mathbb{B}_{n}$.

Proof. It follows from Lemma 22 that $T_{u}$ commutes with $T_{f}$ if and only if

$$
T_{\xi^{k}} T_{u}=T_{u} T_{\xi^{k}}
$$

for all $k \in \mathbb{Z}^{n}$. Suppose that $k=\beta-\gamma$, where $\beta$ and $\gamma$ are two multi-indexes such that $\beta \perp \gamma$; then, by Theorem 21, the above equation is equivalent to

$$
|\beta|=|\gamma| \text { or } f_{k}=0 .
$$

Obviously, (96) is equivalent to

$$
\left(\xi^{k} f_{k}\right)\left(e^{i \theta} z\right)=\left(\xi^{k} f_{k}\right)(z), \quad \forall \theta \in \mathbb{R}, z \in \mathbb{B}_{n} .
$$

Therefore, $T_{u}$ commutes with $T_{f}$ if and only if $f\left(e^{i \theta} z\right)=f(z)$ for almost all $\theta \in \mathbb{R}$ and $z \in \mathbb{B}_{n}$.

Remark 24. In the one-dimensional case, a function satisfying $f\left(e^{i \theta} z\right)=f(z)$ is exactly a radial function, so this corollary coincides with Theorem 6 of [27].

There are lots of examples of functions of the form $\xi^{\beta} u$, which are the symbols of commuting Toeplitz operators (see [27]), but the following theorem will show that two Toeplitz operators with quasihomogeneous symbols of degrees $\beta$ and $-\gamma$, respectively, commute only in the trivial case.

Theorem 25. Suppose that $\beta$ and $\gamma$ are two nonzero multiindexes, and let $u$ and $v$ be two bounded radial functions on $\mathbb{B}_{n}$. If

$$
T_{\xi^{\beta} u} T_{\bar{\xi}^{\gamma} v}=T_{\bar{\xi}^{v} v^{v}} T_{\xi^{\beta} u},
$$

then, $u=0$ or $v=0$.

Proof. For any multi-index $\alpha$, it follows from Lemma 20 that if $\alpha \geq \gamma$, then

$$
\begin{aligned}
T_{\xi^{\beta} u} T_{\bar{\xi}^{\gamma} v} z^{\alpha} \\
=(4 \alpha !(|\alpha|-|\gamma|+n-1) ! \widehat{u}(2 n+2|\alpha|+|\beta|-2|\gamma|) \\
\left.\quad \times \widehat{v}(2 n+2|\alpha|-|\gamma|) z^{\alpha+\beta-\gamma}\right) \\
\quad \times((|\alpha|+|\beta|-|\gamma|)(|\alpha|-|\gamma|)(|\alpha|+n-1) !(\alpha-\gamma) !)^{-1},
\end{aligned}
$$

and, if $\alpha \nsucceq \gamma$, then $T_{\xi^{\beta} u} T_{\bar{\xi}^{\gamma} v} z^{\alpha}=0$.
Similarly, if $\alpha+\beta \succeq \gamma$, then

$$
\begin{aligned}
& T_{\bar{\xi}^{\gamma} v} T_{\xi^{\beta} u} z^{\alpha} \\
&=( 4(\alpha+\beta) !(|\alpha|+|\beta|-|\gamma|+n-1) ! \\
&\left.\times \widehat{u}(2 n+2|\alpha|+|\beta|) \widehat{v}(2 n+2|\alpha|+2|\beta|-|\gamma|) z^{\alpha+\beta-\gamma}\right) \\
& \times((|\alpha|+|\beta|)(|\alpha|+|\beta|-|\gamma|) \\
&\times(|\alpha|+|\beta|+n-1) !(\alpha+\beta-\gamma) !)^{-1},
\end{aligned}
$$

and, if $\alpha+\beta \nsucceq \gamma$, then $T_{\bar{\xi}^{\gamma} v} T_{\xi^{\beta} u} z^{\alpha}=0$.

For two nonzero multi-indexes $\beta$ and $\gamma$, to prove this theorem, we need to consider two cases.

Case 1. Suppose that $\beta_{i_{0}} \neq 0$ and $\gamma \neq 0$ for some $i_{0} \in\{1, \ldots, n\}$. If $T_{\xi \beta u}$ and $T_{\xi^{\gamma_{v}}}$ commute, then

(a) $\widehat{u}(2 n+2|\alpha|+|\beta|) \widehat{v}(2 n+2|\alpha|+2|\beta|-|\gamma|)=0$, if $\alpha+\beta \succeq \gamma$ and $\alpha \nsucceq \gamma$;

(b) $\widehat{u}(2 n+2|\alpha|+|\beta|) \widehat{v}(2 n+2|\alpha|+2|\beta|-|\gamma|)=C_{\alpha} \widehat{u}(2 n+$ $2|\alpha|+|\beta|-2|\gamma|) \widehat{v}(2 n+2|\alpha|-|\gamma|)$, if $\alpha \geq \gamma$,

where

$$
\begin{aligned}
C_{\alpha}= & (\alpha !(|\alpha-| \gamma|+n-1|) !(|\alpha|+|\beta|+n-1) !(\alpha+\beta-\gamma) !) \\
& \times((\alpha+\beta) !(|\alpha|+|\beta|-|\gamma|+n-1) ! \\
& \times(|\alpha|+n-1) !(|\alpha|-|\gamma|)(\alpha-\gamma) !)^{-1}
\end{aligned}
$$

Consider the multi-index $\alpha^{\prime}=\left(\gamma_{1}, \ldots, \gamma_{i_{0}-1}, \gamma_{i_{0}}-\right.$ $\left.1, \gamma_{i_{0}+1}, \ldots, \gamma_{n}\right)$; then $\alpha_{i_{0}}^{\prime}<\gamma_{i_{0}}$ and $\alpha^{\prime}+\beta \succeq \gamma$, since $\beta_{i_{0}} \neq 0$ and $\gamma_{i_{0}} \neq 0$. Denote $a_{0}=\left|\alpha^{\prime}\right|$; then, it follows from (a) that

$$
\widehat{u}\left(2 n+2\left|a_{0}\right|+|\beta|\right) \widehat{v}\left(2 n+2\left|a_{0}\right|+2|\beta|-|\gamma|\right)=0 .
$$

If $\widehat{u}\left(2 n+2 a_{0}+|\beta|\right)=0$, we will let $a_{1}=a_{0}+|\gamma|$; then, a direct calculation from (b) shows that

$$
\widehat{u}\left(2 n+2\left|a_{1}\right|+|\beta|\right) \widehat{v}\left(2 n+2\left|a_{1}\right|+2|\beta|-|\gamma|\right)=0 .
$$

So we can find a sequence $\left\{a_{m}\right\}_{m \in \mathbb{N}}$, which is defined by $a_{m}+$ $1=a_{m}+|s|$ or $a_{m}+|p|$, such that

$$
\widehat{u}\left(2 n+2\left|a_{m}\right|+|\beta|\right) \widehat{v}\left(2 n+2\left|a_{m}\right|+2|\beta|-|\gamma|\right)=0 .
$$

It is clear that $\sum_{m \in \mathbb{N}}\left(1 / a_{m}\right)=\infty$. Let $E_{1}=\{m \in \mathbb{N}: \widehat{u}(2 n+$ $\left.\left.2 a_{m}+|\beta|\right)=0\right\}$ and $E_{2}=\left\{m \in \mathbb{N}: \widehat{v}\left(2 n+2\left|a_{m}\right|+2|\beta|-|\gamma|\right)=0\right\}$. Since

$$
\sum_{m \in \mathbb{N}} \frac{1}{a_{m}} \leq \sum_{m \in E_{1}} \frac{1}{a_{m}}+\sum_{m \in E_{2}} \frac{1}{a_{m}},
$$

we know that at least one of the series $\sum_{m \in E_{1}}\left(1 / a_{m}\right)$ and $\sum_{m \in E_{2}}\left(1 / a_{m}\right)$ diverges; then it follows from Remark 9 that $u=0$ or $v=0$.

Case 2. Suppose that either $\beta_{i}=0$ or $\gamma_{i}=0$ for all $i \epsilon$ $\{1, \ldots, n\}$. Without loss of generality, we can also assume that 
$|\gamma| \geq|\beta|>0$. Obviously, for any multi-index $\alpha \geq \gamma$, the fact that either $\beta_{i}=0$ or $\gamma_{i}=0$ for all $i \in\{1, \ldots, n\}$ implies

$$
\alpha !(\alpha+\beta-\gamma) !=(\alpha+\beta) !(\alpha-\gamma) !
$$

Thus, if $T_{\xi^{\beta} u}$ and $T_{\bar{\xi}^{\gamma} v}$ commute, it follows from (b) that

$$
\begin{aligned}
& (\widehat{u}(2 n+2|\alpha|+|\beta|-2|\gamma|) \hat{v}(2 n+2|\alpha|-|\gamma|)) \\
& \times((|\alpha|+|\beta|-|\gamma|)(|\alpha|-|\gamma|) \\
& \quad \times(|\alpha|+n-|\gamma|) \cdots(|\alpha|+n-|\gamma|+|\gamma|-1))^{-1} \\
& =(\widehat{u}(2 n+2|\alpha|+|\beta|) \widehat{v}(2 n+2|\alpha|+2|\beta|-|\gamma|)) \\
& \times((|\alpha|+|\beta|-|\gamma|)(|\alpha|+|\beta|) \\
& \quad \times(|\alpha|+|\beta|+n-|\gamma|) \cdots \\
& (|\alpha|+|\beta|+n-|\gamma|+|\gamma|-1))^{-1} .
\end{aligned}
$$

As in the proof of Theorem 21, the above equation implies that

$$
\begin{aligned}
& \frac{\widehat{u}(2 z+|\beta|) \widehat{v}(2 z+|\gamma|)}{(z-n)(z+|\beta|-n) z \cdots(z+|\gamma|-1)} \\
& =(\widehat{u}(2 z+|\beta|+2|\gamma|) \hat{v}(2 z+2|\beta|+|\gamma|)) \\
& \quad \times((z+|\beta|-n)(z-n+|\beta|+|\gamma|) \\
& \quad \times(z+|\beta|) \cdots(z+|\beta|+|\gamma|-1))^{-1}
\end{aligned}
$$

for $z \in\{z: \operatorname{Re}(z)>n\}$. Let

$$
f(z)=\frac{\widehat{v}(2 z+|\gamma|)}{(z-n) z \cdots(z+|\gamma|-1)}, \quad g(z)=\frac{\widehat{u}(2 z+|\beta|)}{(z+|\beta|-n)} ;
$$

thus, the above equation can be written as

$$
f(z) g(z)=f(z+|\beta|) g(z+|\gamma|) .
$$

Next, denote

$$
\begin{aligned}
H(z)= & f(z) f(z+1) \cdots f(z+|\beta|-1) \\
& \times g(z) g(z+1) \cdots g(z+|\gamma|-1) .
\end{aligned}
$$

Obviously, $H(z)$ is analytic on $\{z: \operatorname{Re}(z)>n\}$. It follows from (110) and $|\gamma| \geq|\beta|>0$ that

$$
\begin{aligned}
H(z)= & f(z+|\beta|) g(z+|\gamma|) \cdots f(z+|\beta|-1+|\beta|) \\
& \times g(z+|\beta|-1+|\gamma|) \\
& \times g(z+|\beta|) \cdots g(z+|\gamma|-1) \\
= & f(z+|\beta|) f(z+|\beta|+1) \cdots f(z+2|\beta|-1) \\
& \times g(z+|\beta|) g(z+|\beta|+1) \cdots g(z+|\beta|+|\gamma|-1) \\
= & H(z+|\beta|),
\end{aligned}
$$

which implies that $H(z)$ is a periodic function with period $|\beta|$ on $\{z: \operatorname{Re}(z)>n\}$. Thus, the function $H(z)$ can be extended to whole plane $\mathbb{C}$, so we can think of the function $H(z)$ as an entire function. By the definition of the Mellin transform, we get

$$
\begin{aligned}
|H(z)|= & \mid f(z) f(z+1) \cdots f(z+|\beta|-1) g(z) \\
& \times g(z+1) \cdots g(z+|\gamma|-1) \mid \\
\leq & \|u\|_{\infty}^{|\beta|}\|v\|_{\infty}^{|\gamma|} \times\left|\frac{1}{(z-n) \cdots(z+|\beta|-n-1)}\right| \\
& \times\left|\frac{1}{(z+|\beta|-n-1) \cdots(z+|\beta|+|\gamma|-n-1)}\right| \\
& \times \mid \int_{0}^{1} t^{2 z+|\gamma|-1} d t \cdots \int_{0}^{1} t^{2 z+2(|\beta|-1)+|\gamma|-1} d t \\
& \times \frac{\times \int_{0}^{1} t^{2 z+|\beta|-1} d t \cdots \int_{0}^{1} t^{2 z+2(|\gamma|-1)+|\beta|-1} d t \mid}{z \cdots(z+|\gamma|-1)} \cdots \\
= & O \frac{1}{(z+|\beta|-1) \cdots(z+|\beta|+|\gamma|-2)} \\
& \left.\frac{1}{-|\beta||\gamma|-2(|\beta|+|\gamma|)}\right) .
\end{aligned}
$$

Noting that $-|\beta||\gamma|-2(|\beta|+|\gamma|)<0$, we obtain $H(z)=0$, which implies $u=0$ or $v=0$.

\section{Conflict of Interests}

The authors declare that there is no conflict of interests regarding the publication of this paper.

\section{Acknowledgments}

Jin Xia was supported by the China NNSF Grant 11301101, Xiaofeng Wang was supported by the Guangzhou Higher Education Science and Technology Projection no. 2012A018, and Guangfu Cao was supported by the China NNSF Grant 11271092 .

\section{References}

[1] D. Alpay and H. T. Kaptanoğlu, "Toeplitz operators on Arveson and Dirichlet spaces," Integral Equations and Operator Theory, vol. 58, no. 1, pp. 1-33, 2007.

[2] K. Chailuek and B. C. Hall, "Toeplitz operators on generalized Bergman spaces," Integral Equations and Operator Theory, vol. 66, no. 1, pp. 53-77, 2010.

[3] R. Rochberg and Z. J. Wu, "Toeplitz operators on Dirichlet spaces," Integral Equations and Operator Theory, vol. 15, no. 2, pp. 325-342, 1992.

[4] Z. J. Wu, "Hankel and Toeplitz operators on Dirichlet spaces," Integral Equations and Operator Theory, vol. 15, no. 3, pp. 503$525,1992$. 
[5] Y. F. Lu and S. H. Sun, "Toeplitz operators on Dirichlet spaces," Acta Mathematica Sinica (English Series), vol. 17, no. 4, pp. 643648, 2001.

[6] R. G. Douglas, Banach Algebraic Techniques in Operators Theory, vol. 128, Springer, New York, NY, USA, 1971.

[7] A. M. Davie and N. P. Jewell, "Toeplitz operators in several complex variables," Journal of Functional Analysis, vol. 26, no. 4, pp. 356-368, 1977.

[8] J. Miao and D. Zheng, "Compact operators on Bergman spaces," Integral Equations and Operator Theory, vol. 48, no. 1, pp. 61-79, 2004.

[9] N. Zorboska, "Toeplitz operators with BMO symbols and the Berezin transform," International Journal of Mathematics and Mathematical Sciences, no. 46, pp. 2929-2945, 2003.

[10] J. A. Cima and Z. Cuckovic, "Compact Toeplitz operators with unbounded symbols," Journal of Operator Theory, vol. 53, no. 2, pp. 431-440, 2005.

[11] G. F. Cao, "Toeplitz operators with unbounded symbols of several complex variables," Journal of Mathematical Analysis and Applications, vol. 339, no. 2, pp. 1277-1285, 2008.

[12] K. Zhu, Spaces of Holomorphic Functions in the Unit Ball, Springer, New York, NY, USA, 2005.

[13] W. Rudin, Function Theory in the Unit Ball of $\mathbb{C}^{n}$, Springer, New York, NY, USA, 1980.

[14] A. Brown and P. R. Halmos, "Algebraic properties of Toeplitz operators," Journal für die Reine und Angewandte Mathematik, vol. 213, pp. 89-102, 1963.

[15] P. Ahern and Z. Cuckovic, "A theorem of Brown-Halmos type for Bergman space Toeplitz operators," Journal of Functional Analysis, vol. 187, no. 1, pp. 200-210, 2001.

[16] S. Axler and Z. Cuckovic, "Commuting Toeplitz operators with harmonic symbols," Integral Equations and Operator Theory, vol. 14, no. 1, pp. 1-12, 1991.

[17] I. Louhichi, E. Strouse, and L. Zakariasy, "Products of Toeplitz operators on the Bergman space," Integral Equations and Operator Theory, vol. 54, no. 4, pp. 525-539, 2006.

[18] I. Louhichi and L. Zakariasy, "On Toeplitz operators with quasihomogeneous symbols," Archiv der Mathematik, vol. 85, no. 3, pp. 248-257, 2005.

[19] D. Zheng, "Commuting Toeplitz operators with pluriharmonic symbols," Transactions of the American Mathematical Society, vol. 350, no. 4, pp. 1595-1618, 1998.

[20] R. Quiroga-Barranco and N. Vasilevski, "Commutative $C^{*}$ algebras of Toeplitz operators on the unit ball, I. Bargmann-type transforms and spectral representations of Toeplitz operators," Integral Equations and Operator Theory, vol. 59, no. 3, pp. 379419, 2007.

[21] R. Quiroga-Barranco and N. Vasilevski, "Commutative $C^{*}$ algebras of Toeplitz operators on the unit ball, II. Geometry of the level sets of symbols," Integral Equations and Operator Theory, vol. 60, no. 1, pp. 89-132, 2008.

[22] Z.-H. Zhou and X.-T. Dong, "Algebraic properties of Toeplitz operators with radial symbols on the Bergman space of the unit ball," Integral Equations and Operator Theory, vol. 64, no. 1, pp. 137-154, 2009.

[23] R. Remmert, Classical Topics in Complex Function Theory, vol. 172 of Graduate Texts in Mathematics, Springer, New York, NY, USA, 1998.

[24] S. Grudsky, A. Karapetyants, and N. Vasilevski, "Toeplitz operators on the unit ball in $\mathbb{C}^{n}$ with radial symbols," Journal of Operator Theory, vol. 49, no. 2, pp. 325-346, 2003.
[25] B. Choe and H. Koo, "Zero products of Toeplitz operators with harmonic symbols," Journal of Functional Analysis, vol. 233, no. 2, pp. 307-334, 2006.

[26] L. Zakariasy, "The rank of Hankel operators on harmonic Bergman spaces," Proceedings of the American Mathematical Society, vol. 131, no. 4, pp. 1177-1180, 2003.

[27] Z. Cuckovic and N. V. Rao, "Mellin transform, monomial symbols, and commuting Toeplitz operators," Journal of Functional Analysis, vol. 154, no. 1, pp. 195-214, 1998. 


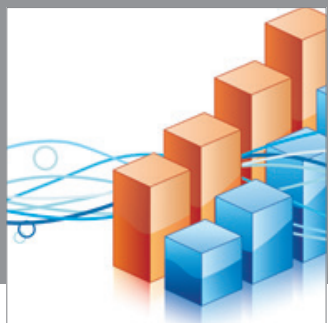

Advances in

Operations Research

mansans

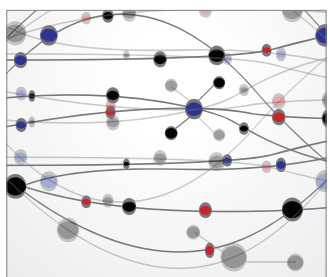

The Scientific World Journal
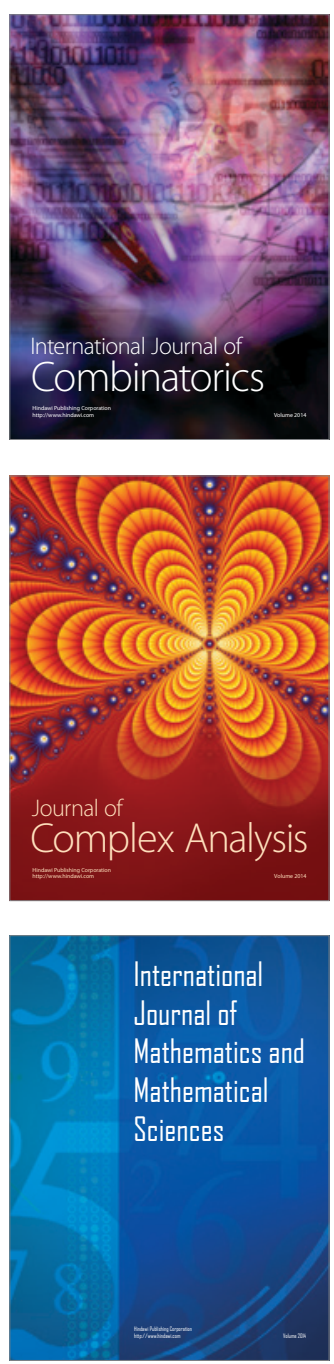
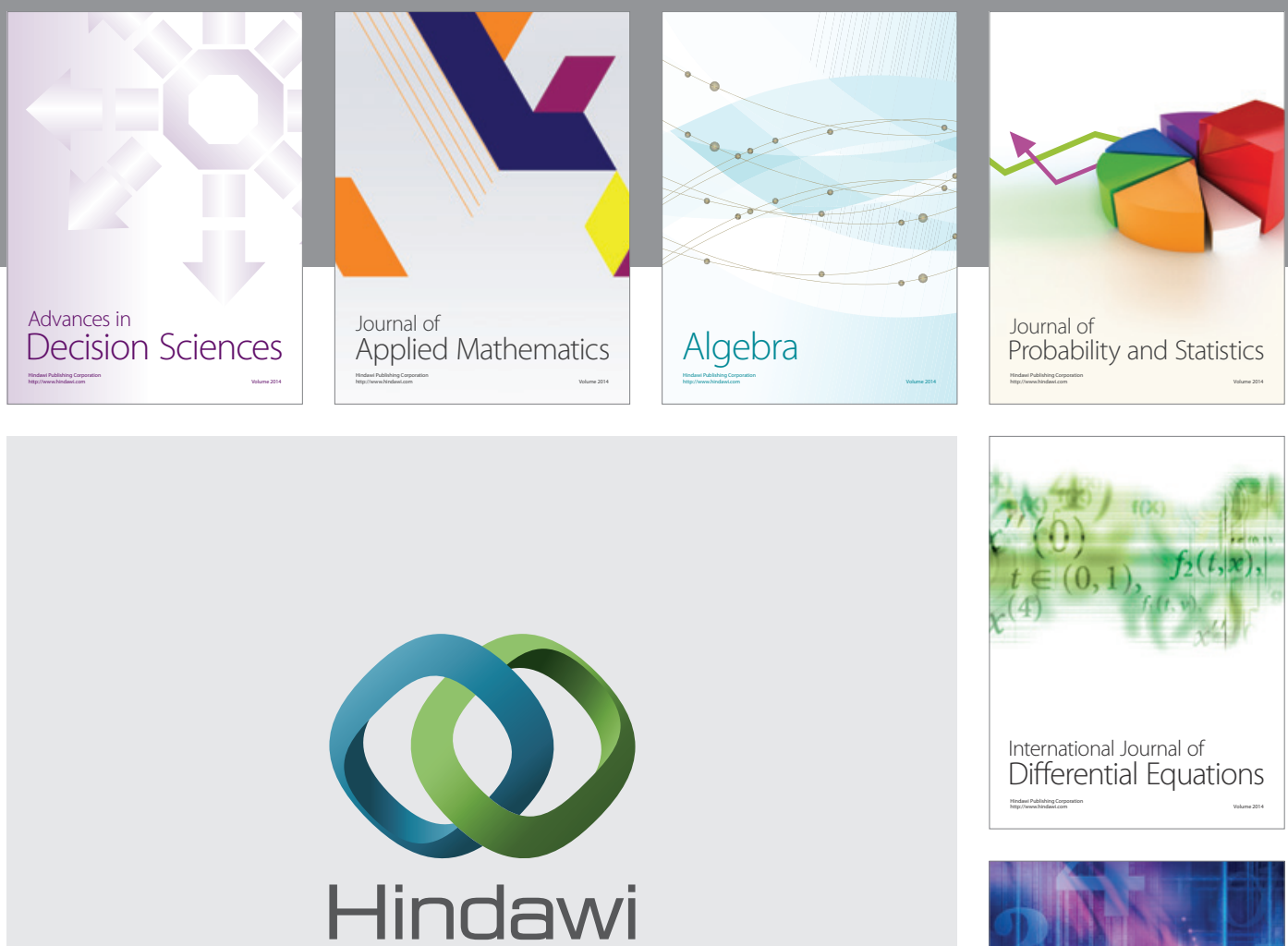

Submit your manuscripts at http://www.hindawi.com
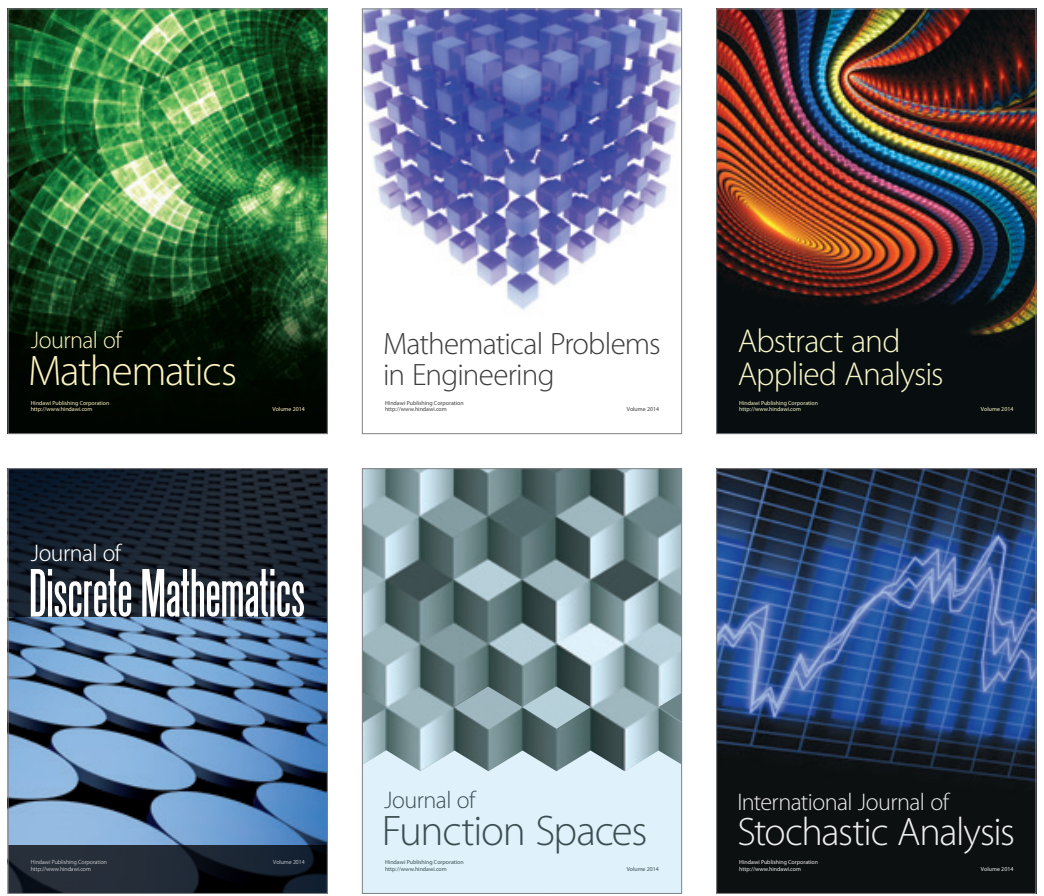

Journal of

Function Spaces

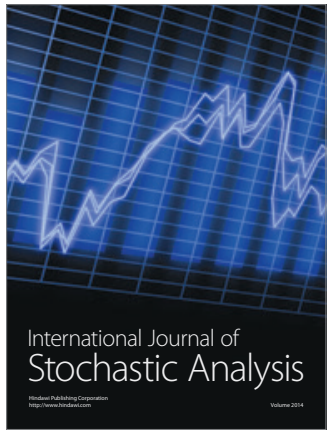

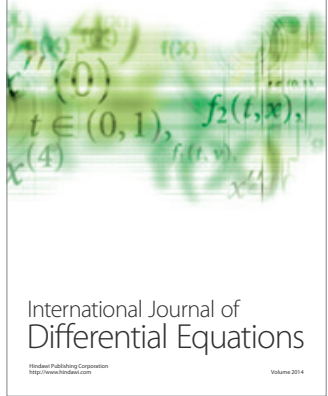
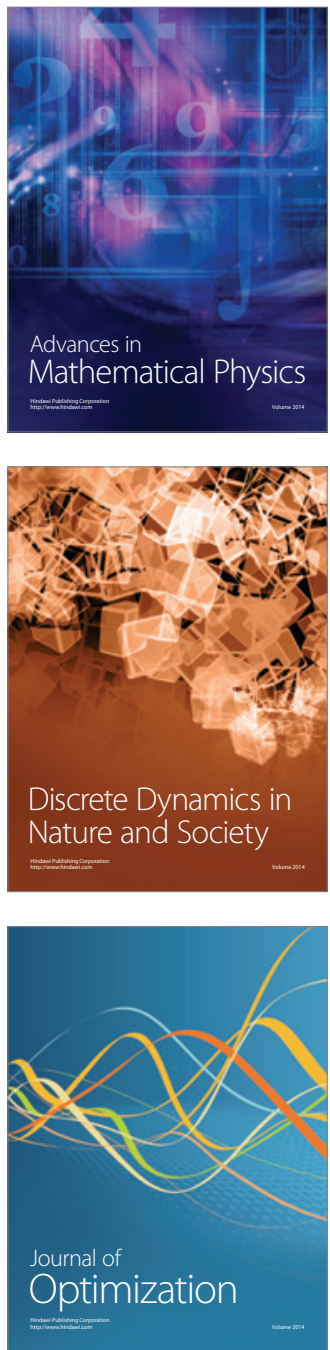